\title{
Intrinsic and Experimental Quasiparticle Recombination Times in Superconducting Films
}

\author{
W. Eisenmenger, K. Laßmann, H. J. Trumpp, and R. Krauß \\ Universität Stuttgart, Physikalisches Institut, D-7000 Stuttgart, Fed. Rep. Germany
}

Received 13 September 1976/Accepted 30 September 1976

\begin{abstract}
Experimental quasiparticle recombination lifetime data for superconducting $\mathrm{Al}$, $\mathrm{Sn}$, and $\mathrm{Pb}$ films are compared with calculations based on a ray acoustic model taking account of the film thickness dependence of the reabsorption of recombination phonons. Information on the true or intrinsic quasiparticle recombination lifetime obtained from these and other data is discussed.
\end{abstract}

PACS Code: 74

The apparent recombination decay time $\tau_{\text {eff }}$ of quasiparticles in superconducting films depends strongly on the reabsorption of phonons emitted in recombination. This influence has been treated in terms of a ray acoustical model [1] for phonon absorption and transmission to the film substrate. In the present article we compare this model with available experimental data. In Sec. 1 we discuss the results of the model calculation, in Sec. 2 possibilities of estimating the important quantity $A_{w}$, the phonon reabsorption mean free path. In Sec. 3 experimental $\tau_{\text {eff }}$ data for $\mathrm{Al}, \mathrm{Sn}$, and $\mathrm{Pb}$ are compared with calculation, together with a discussion of presently available $\tau_{R}$ data.

\section{Results of the Phonon Trapping Model}

The influence of phonon reabsorption can be discussed in terms of three physically significant ranges of the thickness dependence of $\tau_{\text {eff. }}$ For Range 1 with $d \ll \Lambda_{w}$ (d: film thickness, $A_{w}$ : phonon reabsorption mean free path) phonon-quasiparticle scattering as a loss process in the film volume can be neglected and the influence of reabsorption is expressed by

$\frac{1}{\tau_{\text {eff }}}=\frac{1-\cos \varphi_{\max 1}}{\tau_{R l}}+\frac{1-\cos \varphi_{\operatorname{maxt}}}{\tau_{R t}}$.

In this limit phonon reabsorption is determined by the limiting angles $\varphi_{\max 1, \mathrm{t}}$ of the total phonon reflection at the film substrate boundary. The indices $I$ and $t$ refer to longitudinal and transverse phonons, respectively. $\tau_{R l}$ and $\tau_{R t}$ are the intrinsic quasiparticle decay time constants for longitudinal and transverse recombination phonon emission without reabsorption by pair-breaking. Equation (1) indicates that the film thickness $d$ and the phonon transmission coefficients for angles $|\varphi|<\varphi_{\max }$ have no influence on $\tau_{\text {eff }}$.

For Range 2 with $d>\Lambda_{w}$ the calculation [1] results in a linear dependence of $\tau_{\text {eff }}$ on $d$

$\frac{1}{\tau_{\text {eff }}}=\frac{1}{d} \cdot \frac{N_{\omega T}}{N_{T}} \cdot\left[\frac{\bar{T}_{t}}{c_{t}^{2}}+\frac{1}{2} \frac{\bar{T}_{l}}{c_{l}^{2}}\right] /\left(\frac{1}{c_{t}^{3}}+\frac{1}{2 c_{l}^{3}}\right)$

$N_{T}=2 N(0)[2 \pi \Delta(T) k T]^{1 / 2} \exp [-\Delta(T) / k T]$ density of thermally excited quasiparticles with $N(0)$ being the density of Bloch states at $E_{F}$.

$$
\begin{aligned}
N_{\omega T} & =N_{\omega l T}+N_{\omega t T} \\
& =\left(\frac{1}{c_{l}^{3}}+\frac{2}{c_{t}^{3}}\right) \cdot \frac{2 \Delta^{2} k T}{\pi^{2} \hbar^{3}} \cdot\left(1+2 \frac{k T}{2 \Delta}\right) \cdot \exp \left(\frac{-2 \Delta}{k T}\right)
\end{aligned}
$$

density of thermally excited longitudinal and transverse phonons with energy exceeding $2 \Delta$, where $c_{z}$ denotes the longitudinal sound velocity and $c_{t}$ the transverse sound velocity.

$\bar{T}_{l, t}=\int_{0}^{\pi / 2} d \varphi \sin 2 \varphi T_{l, t}(\varphi)$ 
are the average values of the angle dependent film substrate boundary transmission coefficients $T_{i, t}(\varphi)$ for longitudinal and transverse phonons, respectively. In (2) again phonon losses within the film volume are not contained. An estimate [1] of the influence of the dominant loss processes by phonon-quasiparticle scattering shows this approximation to be correct for $T<0.3 T_{c}$ and $\Lambda_{w}<d<10 \Lambda_{w}$. With further increased film thickness $d \gg A_{w}$ and $T>0.5 T_{c}$ phonon decay processes within the film volume lead to Range 3 with comparatively small $2 \Delta$-phonon transmission across the film-substrate boundary and dominant volume decay of phonons. $\tau_{\text {eff }}$ in this range approaches an upper limiting value with no further film thickness dependence resulting in

$\frac{1}{\tau_{\text {eff }}}=\frac{1}{\tau_{R l}\left(1+\Lambda_{v l} / \Lambda_{w l}\right)}+\frac{1}{\tau_{R t}\left(1+\Lambda_{v t} / \Lambda_{w t}\right)}$

where $\Lambda_{v l, t}$ denotes the mean free path for longitudinal and transverse phonons by volume decay processes without by pairbreaking, and $\Lambda_{w l, t}$ the mean free path for longitudinal and transverse phonons by pairbreaking.

Equation (3) corresponds directly to the results of Rothwarf and Taylor [2].

Since information on $A_{w}$, the phonon reabsorption mean free path is of importance in distinguishing between Ranges 1,2 , and 3, we will first discuss the relation between $A_{w}$ and $\tau_{R}$ resulting from the detailed quasiparticle-phonon balance and the possibility of estimating $\Lambda_{w}$ and $\tau_{R}$ from ultrasonic absorption data.

\section{Recombination Time $\tau_{R}$, Phonon Reabsorption Mean Free Path $\boldsymbol{A}_{w}$ and Ultrasonic Absorption}

The recombination time $\tau_{R}$ can be calculated from the mean free path $A_{w}$ for phonon reabsorption, and vice versa, using the detailed balance relation $[1,2]$. In order to check $\tau_{R}$ values, as derived or estimated from $\tau_{\text {eff }}$ measurements, or the amount of phonon trapping, experimental information on $A_{w}$-if not otherwise determined- appears possible by extrapolating ultrasonic absorption data. Taking account of both phonon modes the detailed balance relation $[1,2]$ together with

$\Lambda_{w l, t}=c_{l, t} 4 \cdot \tau_{w l, t}$,

where $\tau_{w l, t}$ is the longitudinal or transverse phonon lifetime for reabsorption by Cooper-pair breaking, results in

$$
\begin{gathered}
\frac{1}{\tau_{R}}=\frac{1}{\tau_{R l}}+\frac{1}{\tau_{R t}}=\frac{4}{N_{T}} \cdot \frac{N_{\omega T}}{\tau_{w}} \\
\tau_{R l, t}=\frac{N_{T}}{c_{l, t}} \frac{A_{w l, t}}{N_{\omega l, t T}}=\frac{N_{T} \cdot \tau_{w l, t}}{N_{\omega l, t T} \cdot c_{l, t} 4}
\end{gathered}
$$

with $\tau_{R l, t}^{-1}$ being the recombination rate with longitudinal or transverse phonon emission,

$\tau_{w l, t}$ the longitudinal or transverse $2 \Delta$-phonon lifetime, and

$\tau_{w}$ the average $2 \Delta$-phonon lifetime.

For a linear frequency dependence of the electronic part of the ultrasonic absorption $\alpha$, as valid [3] (for longitudinal phonons) in the limit $q \cdot l>1$ ( $q$ : phonon wave number, $l$ : electron mean free path), the reabsorption mean free phonon path can be expressed by

$\Lambda_{w l, t}=\frac{4.34 h}{1.57 \cdot K_{l, t} \cdot 2 A(0)}$

with

$K_{l, t}=\frac{\alpha_{l, t}}{v}\left[d \mathrm{~B} \mathrm{~cm}^{-1} \mathrm{~s}\right]$

and $h$ being Planck's constant and $v$ the frequency. The factor 4.34 in the numerator of (5) relates the mean free phonon path to the intensity decay factor $e^{-1}$ of the corresponding sound wave, while the denominator constant of 1.57 refers [4] to the ratio of $\alpha_{s} / \alpha_{n}=1.57$ at $T / T_{c}<0.5$ for the phonons of energy equal to $2 \Delta$ where

$\alpha_{n}$ is the normal conductor ultrasonic absorption constant, and

$\alpha_{s}$ the superconductor ultrasonic absorption constant. From (4), (5), (6) together with $N_{T}, N_{\omega l T}, N_{\omega t T}$ we obtain

$$
\begin{aligned}
\frac{1}{\tau_{R}}= & \frac{18.37 \Delta^{3} \sqrt{\pi}}{h^{4} N(0)} \sqrt{\frac{k T}{2 \Delta}} \\
& \cdot\left(1+2 \frac{k T}{2 \Delta}+\cdots\right)\left(\frac{K_{l}}{c_{l}^{2}}+\frac{K_{t}}{c_{t}^{2}}\right) \exp \left(\frac{-\Delta}{k T}\right) .
\end{aligned}
$$

Equation (7) relates $\tau_{R}$ to the constants $K_{l}$ and $K_{t}$ which can be obtained from ultrasonic absorption data in the $\mathrm{MHz}$ frequency range according to (6). Comparing (7) with theoretical results [5-7] and taking account only of normal processes agreement is found with respect to the temperature dependence of $\tau_{R}$ and also with respect to the energy gap dependence $\tau_{R} \sim \Delta^{-3}$ for constant $k T / \Delta$. 
The linear frequency dependence of the electronic part of ultrasonic absorption both for longitudinal and transverse phonons expressed by (6) holds for normal processes in the range $q \cdot l>1$ (see [8]). In ultrasonic measurements with high-purity single crystals and also in clean metal superconducting tunneling junctions the condition $q \cdot l>1$ can easily be fulfilled. In determining the deformation-potential part of ultrasonic absorption the longitudinal wave contribution is directly obtained from the total electronic attenuation while in the evaluation of the transverse wave contribution electronic absorption in the $\mathrm{MHz}$ frequency range by electromagnetic interaction and phonon collision drag has to be subtracted from the total absorption [8]. These contributions are comparatively small in the high-frequency range of $2 \Delta$-photons [ 3 ], in contrast to possible deformation-potential interaction via Umklapp-processes. Thus, an extrapolation of the ultrasonic $\alpha_{t}$ may become difficult. Umklapp-processes will also modify the extrapolation of $\alpha_{l}$. Furthermore, it has to be noted that for $K_{l}$ and $K_{t}$, averages of $\alpha_{l, t}$ over different crystal orientations must be used. Typical values of $K_{l, t}$ determined from ultrasonic absorption in $\mathrm{Al}, \mathrm{Sn}$, and $\mathrm{Pb}$ range from 0.2 to 0.5 $\left[\mathrm{dB} \mathrm{cm}^{-1} \mathrm{MHz}^{-1}\right]$. With this in mind $\tau_{R}$ estimates from ultrasonic data by use of (7) provide upper limiting values.

\section{Comparison of Experimental and Theoretical Data for $\tau_{\text {eff }}$ and $\tau_{R}$}

The discussion of $\tau_{\text {eff }}$ measurements in tunneling junctions or films reported by different authors requires information on the substrate material and crystal orientation as well as knowledge of whether the sample has been in direct contact with liquid ${ }^{3} \mathrm{He}$ or ${ }^{4} \mathrm{He}$ (see [9]). Also for measurements in vacuum, clean surface conditions are imperative $[9,10]$. An additional experimental check of the reliability of the results is provided by the verification of the temperature dependence $\tau_{\text {eff }} \propto \sqrt{T} \exp (\Delta / k T)$ which is not found in the case of magnetic flux trapping or overinjection. Some authors prefer to report their data in terms of $\tau_{R}^{*}=2 \tau_{R}$ and $\tau_{\mathrm{eff}}^{*}=2 \tau_{\mathrm{eff}}$, i.e. "lifetimes" instead of the experimental "decay time" constants [2], as chosen in [1], where $\tau_{\text {eff }}$ has been used corresponding to the directly observable decay in pulse experiments. For comparison with literature data we also use now $\tau_{R}^{*}$ and $\tau_{\text {eff }}^{*}$. For ease of comparison the results of calculation and experiment are listed in Tables 2 and 3.

\subsection{Aluminum}

The steady state measurements of Miller and Dayem [11], Levine and Hsie [12] using Al-I-Al-I-In sandwich structures on $\mathrm{Al}_{2} \mathrm{O}_{3}$ crystals substrates in direct contact with liquid ${ }^{3} \mathrm{He}$ are more difficult to compare with the phonon escape model than the experiments of Gray et al. [13], and Long [14] which have been performed with $\mathrm{Al}-\mathrm{I}-\mathrm{Al}$ junctions on $\mathrm{Al}_{2} \mathrm{O}_{3}$ crystals in vacuum. For the latter sample configuration we used the data listed in Tables 1 and 2 for calculating $\tau_{\text {eff }}$ in the thickness ranges $d \ll \Lambda_{w}$ and $d \geqq A_{w}$ according to (1) and (2) without volume losses, i.e. $\Lambda_{v} \gg \Lambda_{w}$. For Range 1 with sample thickness $d \ll \Lambda$ we obtain from (1) for $\mathrm{Al}$ on $\mathrm{Al}_{2} \mathrm{O}_{3}$

$\frac{1}{\tau_{\mathrm{eff}}}=\frac{0.186}{\tau_{R l}}+\frac{0.142}{\tau_{R t}}$

With $\tau_{R l}>\tau_{R t}$ as to be expected from theory $[7,15]$ we obtain $\tau_{\text {eff }}=7 \cdot \tau_{R}$, i.e. phonon trapping cannot be less than by a factor of 7 for clean and plane $\mathrm{Al}$ junctions on $\mathrm{Al}_{2} \mathrm{O}_{3}$ in vacuum. Surface irregularities can reduce this trapping factor by diffuse phonon scattering [1].

For an estimate $\tau_{R}$ by the detailed balance relation we can use the value $A_{w t}=3500 \AA$, as obtained experimentally by Long [16] for the dominant transverse phonon contribution. Extrapolation of ultrasonic data of David [17] according to (5) and (6) results in average values of $K_{t}=0.8$ and $K_{l}=0.4\left[\mathrm{~dB} \mathrm{~cm}^{-1} \mathrm{MHz}^{-1}\right]$ and $A_{\mathrm{t}}=3600 \AA, \Lambda_{l}=7200 \AA$ for $\Delta=200 \mu \mathrm{eV}$ if the total normal state electronic absorption at $\mathrm{MHz}$ frequencies is taken into account. In view of the mentioned difficulties of this extrapolation the agreement between Long's data for $\alpha_{t}$ and the ultrasonic extrapolation appears satisfactory. In using $\Lambda_{t}=3500 \AA$ and $\Lambda_{l}=7200 \AA$ the $\tau_{R}$ calculation with (4) results in $\tau_{R t}=$ $1.05 \times 10^{-7} \mathrm{~s}, \tau_{R l}=2.7 \times 10^{-6} \mathrm{~s}$ and finally in the intrinsic decay time $\tau_{R}=1.04 \times 10^{-7} \mathrm{~s}$ at $\Delta / k T=4$ and $\Delta=200 \mu \mathrm{eV}$. The intrinsic recombination lifetime amounts to $\tau_{R}^{*}=2.1 \times 10^{-7} \mathrm{~s}$. Since $\tau_{R l} \gg \tau_{R t}$ is confirmed, the thin limit for $\tau_{\text {eff }}$ with a trapping factor of 7 results in $\tau_{\text {eff }}=7.3 \times 10^{-7} \mathrm{~s}$ or $\tau_{\text {eff }}^{*}=1.45 \times 10^{-6} \mathrm{~s}$.

So far the possibility of comparison with experimental data on thin film Al junction is limited. Long [14] reported lifetimes $\tau_{\text {eff }}^{*}=1.4 \mu \mathrm{s}$ and $1.5 \mu \mathrm{s}$ measured by pulse decay with $\mathrm{Al}$ junctions $710 \AA$ and $950 \AA$ thick on $\mathrm{Al}_{2} \mathrm{O}_{3}$, respectively, (with $\Delta=200 \mu \mathrm{eV}$ at $\Delta / k T=4$ ) in agreement with our thin limit calculation. But also shorter lifetimes are observed with other similar samples. In these cases basically a crystalline surface 
structure leading to diffuse phonon scattering could possibly account for a reduction of the limiting trapping factor [1]; also surface overlays of condensed residual gas have a similar effect [1]. In further measurements by Long [14] with samples of $1000 \AA$ thickness with silicon monoxide overlay, lifetimes $\tau_{\text {eff }}^{*}=0.45 \mu$ s were observed, indicating also enhanced phonon escape. Levine and Hsieh [12] reported decay times $\tau_{\text {eff }}=0.2 \mu \mathrm{s}$ at $\Delta=4 k T$ in Al junctions of $700 \AA$ thickness with indium overlay in contact with liquid ${ }^{3} \mathrm{He}$ but without specifying the energy gap. In their sample configuration the In overlay and the ${ }^{3} \mathrm{He}$ contact destroys the limiting angle of total reflection in the Al junction. Consequently, values closer to our calculated intrinsic $\tau_{R}=0.1 \mu$ s or $\tau_{R}^{*}=0.21 \mu$ s are to be expected. The same argument applies to the results of Miller and Dayem [11], having obtained a decay time of $0.6 \mu \mathrm{s}$ at $\Delta=4 k T$ with $\Delta=220 \mu \mathrm{eV}$, the $\mathrm{Al}$ junction thickness amounting to $500 \AA$.

In a recent steady-state experiment Smith and Mochel [18] determined the intrinsic lifetime $\tau_{R}^{*}$ for $900 \AA$ Al films on glass. In this measurement the phonon trapping factor was directly obtained by alternating creation of quasiparticles via single particle tunneling and pairbreaking by phonons. From the different quasiparticle populations for the sample in vacuum or in contact with liquid ${ }^{4} \mathrm{He}$ the phonon trapping factor was evaluated with $8.6 \pm 1.1$ (vacuum condition). Since the acoustic properties of glass are not too far from $\mathrm{Al}_{2} \mathrm{O}_{3}$ this trapping factor shows reasonable. agreement with the calculated thin limit value of 7 for $\mathrm{Al}$ on $\mathrm{Al}_{2} \mathrm{O}_{3}$.

The intrinsic lifetime measured by Smith and Mochel [18] with $\tau_{R}^{*}=1.4 \pm 0.1 \mu \mathrm{s}$ at $\Delta / k T=6$ reduces to $\tau_{R}^{*}=0.154 \mu \mathrm{s}$ at $\Delta / k T=4$ in reasonable agreement with our estimated value of $\tau_{R}^{*}=0.2 \mu \mathrm{s}$.

Finally this number is to be compared with recent lifetime calculations of Kaplan et al. [19] using $\alpha^{2}(\Omega) F(\Omega)$ obtained from theory or tunneling measurements.

For $\mathrm{Al}$ and $\Delta / k T=4$ their data result in $\tau_{R}^{*}=0.88 \mu \mathrm{s}$ about four times larger than our value. From this calculation [19] also the average phonon reabsorption mean free path results with the higher value of $\Lambda_{w}=7500 \AA[20,32]$.

Since most of the experimental data scatter within the limits of $\tau_{R}^{*}=0.21 \mu \mathrm{s}$ (following from $A_{w}=3500 \AA$ ) and the calculated thin limit effective time $\tau_{\text {eff }}^{*}=1.45 \mu \mathrm{s}$ for $\mathrm{Al}$ junctions on $\mathrm{Al}_{2} \mathrm{O}_{3}(\Delta=4 \mathrm{kT}$ and $\Delta=200 \mu \mathrm{eV})$, still further careful experimental work also specifying surface structure and purity remains to be done in this thickness region. From such measurements conclusive information on $\tau_{R}$ and $\Lambda_{w}$ in $\mathrm{Al}$ is expected.

For the film thickness Range 2 with $\alpha>\Lambda_{w}$ and proportionality between $\tau_{\text {eff }}$ and $d$ some experimental data are available by the measurements of Long [14].

Our calculation using the data for $\mathrm{Al}$ on $\mathrm{Al}_{2} \mathrm{O}_{3}$ substrates in vacuum according to (2) with $\Lambda_{v} \gg \Lambda_{w}$ again for $\Delta=4 k T$ and $\Delta=200 \mu \mathrm{eV}$ results in: $\tau_{\text {eff }}^{*}=0.108 \times d /[\mathrm{cm}]$ [s]. Experimentally Long [14] obtained for several measurements in the thickness range $d=1520 \AA$ to $d=3540 \AA$ (reduced to $\Delta=200 \mu \mathrm{eV}$ ) $\tau_{\text {eff }}^{*}=0.087( \pm 9 \%) \cdot d /[\mathrm{cm}][\mathrm{s}]$. The agreement [31] appears satisfactory since the average phonon boundary escape [21] values $\bar{T}_{t}=0.20$ and $\bar{T}_{l}=0.3$ used in the evaluation of (2) are also known only within $10 \%$ accuracy. This gives further support to Long's conclusion that his experimental results between $d=1500 \AA$ and $3500 \AA$ are well described within the linear thickness dependence regime of $\tau_{\text {eff }}^{*}$ on $d$. Apparently the acoustic boundary phonon escape model can be directly applied to evaporated pure $\mathrm{Al}$ films on $\mathrm{Al}_{2} \mathrm{O}_{3}$ and influences of grain boundaries or of the oxide barrier within the film on $2 A$-phonon scattering or propagation are not found. This is in agreement with Kinder's [22] result that the sound velocities in thin Al films as used in our evaluation agree with the velocities measured in bulk polycrystalline material. The linear thickness dependence of $\tau_{\text {eff }}^{*}$ in Long's measurements down to $1500 \AA$ indicates the possibility of diffuse surface scattering [1], i.e. unchanged average value $\bar{T}$ but increased total reflection angles $\varphi_{\max }$; or that in these films the mean free phonon path for transverse waves is smaller than $3500 \AA$. This is consistent with the transverse and longitudinal phonon resonances found by Kinder [22] in $\mathrm{Al}$ phonon detectors of film thickness $620 \AA$ and $920 \AA$ indicating a phonon mean free path in the range between $1000 \AA$ and $3000 \AA$. The observed phonon interference in tunneling junctions of enhanced energy gap by oxygen background evaporation is in agreement with our suggestion that grain boundaries within the film and oxide tunneling barriers are not strong phonon scatterers. Also the results of Welte et al. [23] and Forkel et al. [24] on the high energy phonon escape from Al junctions are consistent with a phonon mean free path at $2 \Delta_{\mathrm{Al}}$ of about $2000 \AA$ and no significant elastic phonon scattering within the film.

In order to resolve the question, whether the linear thickness dependence in the range $1500 \AA$ to $3500 \AA$ in Long's [14] measurements results from diffuse surface scattering of phonons or from $\Lambda_{w}$ being less than 
Table 1. Acoustic data used for the evaluation of (1) and (2)

\begin{tabular}{lccl}
\hline & $\begin{array}{l}\varrho \\
{\left[\mathrm{g} \cdot \mathrm{cm}^{-3}\right]}\end{array}$ & $\begin{array}{l}c_{l} \cdot 10^{5} \\
{\left[\mathrm{~cm} \mathrm{~s}^{-1}\right]}\end{array}$ & $\begin{array}{l}c_{\mathrm{t}} \cdot 10^{5} \\
{\left[\mathrm{~cm} \mathrm{~s}^{-1}\right]}\end{array}$ \\
\hline $\mathrm{Al}$ & 2.72 & $6.4[42]$ & $3.1[42]$ \\
$\mathrm{Sn}$ & 7.3 & $3.32[42]$ & $1.67[42]$ \\
$\mathrm{Pb}$ & 11.8 & $2.35[43]$ & $1.03[43]$ \\
$\mathrm{Al}_{2} \mathrm{O}_{3}$ & 4.0 & 11.0 & 6.04 \\
$c$-axis & & & \\
$\mathrm{Si}$ & 2.35 & 9.35 & 5.2 \\
111 & & & \\
\hline
\end{tabular}

$3500 \AA$, further measurements with specified surface of the films are necessary.

Influences of bulk phonon absorption, i.e. independence of $\tau_{\text {eff }}$ on thickness at large $d$ values in Range 3 so far have not been found. Experimentally this would require the use of thin $\mathrm{Al}$ crystal plates or evaporated films of thickness exceeding $d=10000 \AA$ at temperatures $T>0.5 T_{c}$.

For completeness the calculated results for $\mathrm{Al}$ on $\mathrm{Si}$ with respect to Range 1 and 2 are also introduced in Table 2. Corresponding experimental data are not available. The acoustical data of Si are listed in Table 1.

\subsection{Tin}

Measurements of $\tau_{\text {eff }}$ using pulse methods have been performed for $\mathrm{Sn}$ tunneling junctions on silicon substrates in vacuum and ${ }^{4} \mathrm{He},[9,25]$ and also on $\mathrm{Al}_{2} \mathrm{O}_{3}$ substrates with ${ }^{4} \mathrm{He}$ contact [26] and with additionally evaporated solid nitrogen layers [10]. $\tau_{\text {eff }}$ measurements using optical excitation with $\mathrm{Sn}$ on $\mathrm{SiO}_{2}$ and $\mathrm{Al}_{2} \mathrm{O}_{3}$ in vacuum are reported by Sai-Halász et al. [27] and Parker [28].

For the small film thickness Range $1 d \ll \Lambda_{w}$ we obtain from (1) and the data in Table 1 and 2

$\frac{1}{\tau_{\text {eff }}}=\frac{0.047}{\tau_{R l}}+\frac{0.039}{\tau_{R t}}$ for $\mathrm{Sn}$ on $\mathrm{Al}_{2} \mathrm{O}_{3}$

and

$\frac{1}{\tau_{\text {eff }}}=\frac{0.065}{\tau_{R l}}+\frac{0.053}{\tau_{R t}}$ for $\mathrm{Sn}$ on $\mathrm{Si}$.

With dominating transverse phonon contribution this results in thin limit trapping factors of 25.6 and 18.8 for $\mathrm{Sn}$ on $\mathrm{Al}_{2} \mathrm{O}_{3}$ and $\mathrm{Sn}$ on $\mathrm{Si}$, respectively.

Phonon mean free path values for calculating $\tau_{R}$ range from direct $2 \Delta$ phonon reabsorption average values of $\Lambda_{w} \leqq 700 \AA[29]$ to ultrasonic data [30] extrapolation with average values $\Lambda_{l}=1600 \AA$ and $\Lambda_{t}=2200 \AA$ or $A_{t}=7300 \AA$, accounting for the total transverse absorption or only for the deformation potential contribution, respectively, for the case of normal processes. From these different mean free path values it appears that ultrasonic data extrapolation for transverse phonons to frequencies at $2 \Delta_{\mathrm{Sn}}$ does not describe the experimental limit $\Lambda_{w} \leqq 700 \AA$, this apparently results from dominant Umklapp contributions to phonon

Table 2. Numerical results of the phonon escape model and some experimental data for $\tau_{\text {eff }}$ in thickness Ranges 1 and 2 with various superconductor-substrate combinations

\begin{tabular}{|c|c|c|c|c|c|c|c|}
\hline & \multirow{2}{*}{$\begin{array}{l}\text { Superconductor } \\
\text { Substrate }\end{array}$} & \multicolumn{2}{|l|}{$\mathrm{Al}$} & \multicolumn{2}{|l|}{$\mathrm{Sn}$} & \multicolumn{2}{|l|}{$\mathrm{Pb}$} \\
\hline & & $\mathrm{Al}_{2} \mathrm{O}_{3}$ & $\mathrm{Si}$ & $\mathrm{Al}_{2} \mathrm{O}_{3}$ & $\mathrm{Si}$ & $\mathrm{Al}_{2} \mathrm{O}_{3}$ & $\mathrm{Si}$ \\
\hline $\begin{array}{l}\text { Limiting angle of total } \\
\text { phonon reflection in the } \\
\text { film }\end{array}$ & $\varphi_{\max l}$ & $\begin{array}{l}35.6^{\circ} \\
30.9^{\circ}\end{array}$ & $\begin{array}{l}43.2^{\circ} \\
36.6^{\circ}\end{array}$ & $\begin{array}{l}17.6^{\circ} \\
16.0^{\circ}\end{array}$ & $\begin{array}{l}20.8^{\circ} \\
18.7^{\circ}\end{array}$ & $\begin{array}{r}12.3^{\circ} \\
9.8^{\circ}\end{array}$ & $\begin{array}{l}13.6^{\circ} \\
10.9^{\circ}\end{array}$ \\
\hline Range $1 d \ll A_{w}$ Eq. (1) & $1-\overline{\cos \varphi_{\max l}}$ & 0.19 & $0 . \overline{27}$ & 0.047 & 0.065 & 0.023 & 0.028 \\
\hline$\frac{1}{\tau_{\text {eff }}}=\sum_{l, t} \frac{1-\cos \varphi_{\max l, t}}{\tau_{R l, t}}$ & $1-\cos \varphi_{\max t}$ & 0.14 & 0.20 & 0.039 & 0.053 & 0.015 & 0.018 \\
\hline $\begin{array}{l}\text { phonon trapping factor } A \\
\tau_{\text {eff }}=A \tau_{R}\end{array}$ & $\begin{array}{l}A_{\text {theor }} \\
A_{\text {exp }} \\
\end{array}$ & $\begin{array}{l}7 \\
8.6^{d}\end{array}$ & $\begin{array}{r}5 \\
- \\
\end{array}$ & $\begin{array}{l}25.6 \\
- \\
\end{array}$ & $\begin{array}{l}18.8 \\
- \\
\end{array}$ & $\begin{array}{l}67 \\
- \\
\end{array}$ & $\begin{array}{l}56 \\
- \\
\end{array}$ \\
\hline $\begin{array}{l}\left.\text { Range } 2 d \geqq A_{w} \text { Eq. ( } 2\right) \\
\text { average phonon boundary } \\
\text { transmission factors } \\
\bar{T}_{l, t} \text { and constant of } \\
\text { linear thickness } \\
\text { dependence } B ; \tau_{\text {eff }}=B \cdot d\end{array}$ & $\begin{array}{l}\bar{T}_{l}^{\mathrm{c}} \\
\bar{T}_{t}^{\mathrm{c}} \\
B_{\text {theor }}\left[\mathrm{s} \mathrm{cm}^{-1}\right] \\
B_{\text {exp }}\left[\mathrm{s} \mathrm{cm}^{-1}\right]\end{array}$ & $\begin{array}{l}0.3^{\mathrm{a}} \\
0.2^{\mathrm{a}} \\
0.054 \\
0.044^{\mathrm{e}}\end{array}$ & $\begin{array}{l}0.46 \\
0.34 \\
0.028 \\
-\end{array}$ & $\begin{array}{l}0.084 \\
0.068 \\
4.38 \times 10^{-3} \\
4.2 \times 10^{-3 f}\end{array}$ & $\begin{array}{l}0.12^{\mathrm{b}} \\
0.094^{\mathrm{b}} \\
3.48 \times 10^{-3} \\
3.30 \times 10^{-3} \mathrm{~g}\end{array}$ & $\begin{array}{l}0.043 \\
0.026 \\
1.28 \times 10^{-3} \\
-\end{array}$ & $\begin{array}{l}0.063 \\
0.037 \\
0.88 \times 10^{-3} \\
-\end{array}$ \\
\hline
\end{tabular}

References: ${ }^{\mathrm{a}}[21] ;{ }^{\mathrm{b}}[44] ;{ }^{\mathrm{c}}[45] ;{ }^{\mathrm{d}}[18] ;{ }^{\mathrm{e}}[14] ;{ }^{\mathrm{f}}[28,10,26]$ average, liq. He contact accounted for by a factor of $4 ; \mathrm{e}[9,25]$. 


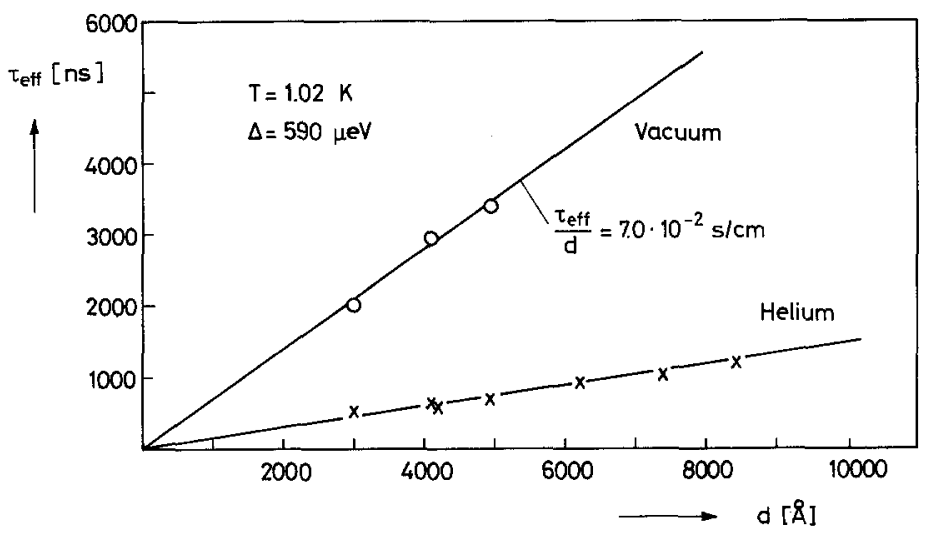

Fig. 1. Experimental recombination lifetime for Sn-junctions on Si-substrates [25] as function of film thickness. Pulse decay measurements in vacuum and also with liquid $\mathrm{He}$ contact. For $T=1.02 \mathrm{~K}$ the vacuum measurement results in $\tau_{\text {eff }} / d=7.0 \times 10^{-2} \mathrm{~s} \cdot \mathrm{cm}^{-1}$. From this follows: $\tau_{\text {eff }} / d=3.3 \times 10^{-3} \mathrm{~s} \cdot \mathrm{cm}^{-1}$ at $T=4 / 4 k=1.71 \mathrm{~K}$ (compare Subsec. 3.2.). The experimental temperature dependence has been verified to follow the $\tau_{\operatorname{eff}} \propto \exp (\Delta / k T)$ law

absorption. With $A_{w t}=700 \AA$ and $A_{w l}=1600 \AA$ the detailed balance equation (4) results in

$\tau_{R t}=6.75 \times 10^{-10} \mathrm{~s}$ and $\tau_{R l}=1.08 \times 10^{-8} \mathrm{~s}$

for $\Delta=4 k T$ and $\Delta=590 \mu \mathrm{eV}$. The transverse phonon contribution again dominating it follows that $\tau_{R}=6.36 \times 10^{-10} \mathrm{~s}$ and $\tau_{R}^{*}=1.27 \times 10^{-9} \mathrm{~s}$. Using the thin limit trapping factors this results in

$\tau_{\text {eff }, \mathrm{Sn} / \mathrm{Al}_{2} \mathrm{O}_{3}}=1.63 \times 10^{-8} \mathrm{~s}$

and

$\tau_{\text {eff }, \mathrm{Sn} / \mathrm{Si}}=1.19 \times 10^{-8} \mathrm{~s}$

for Sn junctions with $d<\Lambda_{w}$ under vacuum conditions on $\mathrm{Al}_{2} \mathrm{O}_{3}$ and $\mathrm{Si}$ substrates, respectively.

Experimental results in this thickness range are available from the measurements of Sai-Halász et al. [27] for $400 \AA \mathrm{Sn}$ films on quartz under vacuum conditions. The observed effective lifetimes reduced to $\Delta / k T=4$ are $\tau_{\text {eff }}^{*}=3.0 \times 10^{-8} \mathrm{~s}$. From the limiting angle of total reflection for $\mathrm{Sn}$ on $\mathrm{SiO}_{2}$ and the dominant transverse phonons we obtain according to (1) a trapping factor of 15 . This results in $\tau_{R}^{*}=2.0 \times 10^{-9} \mathrm{~s}$ from the measurement of Sai-Halász et al. reasonably close to our value $\tau_{\boldsymbol{R}}^{*}=1.27 \times 10^{-9} \mathrm{~s}$. In the theoretical work of Kaplan et al. [19] a somewhat longer lifetime of $\tau_{R}^{*}=4.6 \times 10^{-9} \mathrm{~s}$ at $\Delta / k T=4$ was obtained together with a larger phonon reabsorption mean free path (dominant transverse phonons) of $\Lambda_{w}=1840 \AA[20,32]$. Experiments with $\mathrm{Sn}$ tunneling junctions as phonon generators in contrast show strong reabsorption of relaxation phonons with energy exceeding $2 \Delta$ in accord with $\Lambda_{w}<1000 \AA$.

In most experiments on $\mathrm{Sn}[9,10,25-28,33]$ the film thickness exceeded $1000 \AA$ and we expect a thickness dependence of $\tau_{\text {eff }}$ corresponding to Range 2 with $d>\Lambda_{w}$. Applying (2) with $\Lambda_{v} \gg \Lambda_{w}$ to the acoustical data of $\mathrm{Sn}$ on $\mathrm{Al}_{2} \mathrm{O}_{3}$ and $\mathrm{Si}$ results in

$\tau_{\text {eff }, \mathrm{Sn} / \mathrm{Al}_{2} \mathrm{O}_{3}}=4.38 \times 10^{-3} d /[\mathrm{cm}][\mathrm{s}]$ for $\mathrm{Al}_{2} \mathrm{O}_{3}$ substrates and

$\tau_{\mathrm{eff}, \mathrm{Sn} / \mathrm{Si}}=3.48 \times 10^{-3} d /[\mathrm{cm}][\mathrm{s}]$

for $\mathrm{Si}$ substrates at $\Delta=4 k T$ and $\Delta=590 \mu \mathrm{eV}$. Experimental results by pulse decay measurements under vacuum conditions for $\mathrm{Sn}$ on $\mathrm{Si}[9,25]$, compare Fig. 1, indicate the expected linear dependence of $\tau_{\text {eff }}$ on $d$. From Fig. 1 the average slope of

$\tau_{\text {eff }}=7.0 \times 10^{-2} d /[\mathrm{cm}][\mathrm{s}]$

for $T=1.02 \mathrm{~K}$ and $\Delta=590 \mu \mathrm{eV}$ after reduction to $\Delta=4 k T$ results in

$\tau_{\text {eff }, \mathrm{Sn} / \mathrm{Si}}=3.30 \times 10^{-3} d /[\mathrm{cm}][\mathrm{s}]$

in good agreement with the calculated value $3.48 \times 10^{-3} d /[\mathrm{cm}][\mathrm{s}]$. The total error between calculation and measurement amounts to $\pm 10 \%$ corresponding to the limited accuracy of the calculation of $\bar{T}_{t}$ and $\bar{T}_{l}$ and the finite width of the energy gap.

We note that in this thickness regime the contribution of the longitudinal phonon escape amounts to $13 \%$ of the total phonon escape rate. The phonon trapping factor for the measurement with $d=3000 \AA$ amounts to 140 comparing $\tau_{\text {eff }}$ with $\tau_{R}$ as obtained from $\Lambda_{w}=700 \AA$. Since Fig. 1 shows no deviation from the linear thickness dependence down to $d=3000 \AA$, the conclusion that $\Lambda_{w}$ is smaller than $3000 \AA$ is consistent with $\Lambda_{w}=700 \AA$. In contact with superfluid $\mathrm{He}$ the thickness proportionality of $\tau_{\text {eff }}$ in Fig. 1 is reduced by a factor of 4.6 indicating the strong deviation of the phonon escape $[9,34-36]$ into liquid ${ }^{4} \mathrm{He}$ from the acoustic model. For $\mathrm{Sn}$ on $\mathrm{Al}_{2} \mathrm{O}_{3}$ in contact with liquid $\mathrm{He}$ Parker [28] obtained the experimental value $\tau_{\text {eff }}=1.31 \times 10^{-3} d /[\mathrm{cm}][\mathrm{s}](\Delta=4 k T)$ while pulse decay measurements $[10,26]$ with experimental data 
$d=2300 \AA, \tau_{\text {eff }}=8 \times 10^{-8} \mathrm{~s}$ at $T=1.25 \mathrm{~K}$ result in $\tau_{\text {eff }}=0.8 \times 10^{-3} d /[\mathrm{cm}][\mathrm{s}](\Delta=4 k T)$. The difference of the experimental values exceeds the limits of $\pm 30 \%$, as estimated in [28]. Comparing experiment with our calculated "vacuum value" $\tau_{\text {eff }}=4.38 \times 10^{-3} d /[\mathrm{cm}][\mathrm{s}]$ $(\Delta=4 k T)$ the increase of the phonon escape rate by a factor of 3.3 to 5.5 agrees with the escape rate increase for $\mathrm{Sn}$ on $\mathrm{Si}$ in He. Finally, pulse decay measurements with optical excitation [33] of a Sn tunneling junction $d=3200 \AA$ on glass in ${ }^{4} \mathrm{He}$ contact resulted in $\tau_{\text {eff }}=3.9 \times 10^{-8} \mathrm{~s}(\Delta=4 k T)$. This leads to

$\tau_{\text {eff }}=1.22 \times 10^{-3} d /[\mathrm{cm}][\mathrm{s}]$

in agreement with the above data for $\mathrm{Sn}$ on $\mathrm{Al}_{2} \mathrm{O}_{3}$ in contact with liquid $\mathrm{He}$. This is to be expected since the phonon radiation into the helium bath dominates.

An additional reduction of $\tau_{\mathrm{eff}}$ in the linear thickness range has been observed by condensing nitrogen [10] on $2330 \AA \mathrm{Sn}$ films on $\mathrm{Al}_{2} \mathrm{O}_{3}$ in contact with liquid $\mathrm{He}$. The measured time constant is $\tau_{\text {eff }}=1.8 \times 10^{-7} \mathrm{~s}$ at $1.02 \mathrm{~K}$ and corresponds to a trapping factor of about 13 as compared to $\tau_{R}$ with $A_{w}=700 \AA$ at the same temperature. Evaluation by (2) indicates an increased phonon escape of about $\bar{T}_{t, l}=0.8$ or $80 \%$ by the condensed nitrogen-liquid He contact. Part of this influence is due to the limiting angles of total reflection in Sn increasing to $90^{\circ}$ since the sound velocities in solid $\mathrm{N}_{2}$ and liquid $\mathrm{He}$ are smaller than the sound velocities in Sn. Quantitatively the acoustic model, however, cannot account for the high phonon escape rate as is the case for the Kapitza resistance anomaly of liquid He. In contrast to this the acoustical model even at frequencies of $280 \mathrm{GHz}$ corresponding to $2 \Delta_{\mathrm{S}_{\mathrm{n}}}$ can be applied to the metal-solid boundary as is demonstrated by the close agreement between measurement and calculation for experiments under vacuum conditions.

Finally, also for the case of Sn no influence of volume absorption of phonons has been observed even at trapping factors exceeding 100 , as indicated by the absence of saturation effects at larger $d$ values in the $\tau_{\text {eff }}(d)$ dependence of Fig. 1. This result is of importance for the use of tunneling junctions as phonon generators [37] since the conclusion can be drawn that all recombination phonons leave the junction (under vacuum conditions) without volume losses. Further experiments with increased film thickness and temperature, i.e. in Range 3, are necessary to study the onset of volume losses and the possible application to phonon mean free path determination.

\subsection{Lead}

Although the first experimental and theoretical work $[1,5,15]$ in order to determine $\tau_{R}$ has been concentrated on lead, only a few further measurements [39] have been performed. In the experiments samples in direct contact to liquid $\mathrm{He}$ were used. At $1.44 \mathrm{~K}$ the experimental value was $\tau_{\text {eff }}^{*} \leqq 2.2 \times 10^{-7} \mathrm{~s}$; the theoretical values are $\tau_{R}^{*}=4.3 \times 10^{-8} \mathrm{~s}[5]$ and $\tau_{R}^{*}=1.67 \times 10^{-8} \mathrm{~s}$ [15], both at $1.44 \mathrm{~K}$, the latter also taking account of Umklapp processes. In [39] experiments on films with $1000-2000 \AA$ thickness are reported which result in $\tau_{\text {eff }}$ values exceeding the theoretical result [15] by one order of magnitude.

For comparison of the experimental results with our model the acoustical data of Tables 1 and 2 are introduced in (1). For Range 1 with $d<\Lambda_{w}$ we obtain

$\frac{1}{\tau_{\text {eff }}}=\frac{0.023}{\tau_{R l}}+\frac{0.015}{\tau_{R t}}$ for $\mathrm{Pb}$ on $\mathrm{Al}_{2} \mathrm{O}_{3}$ in vacuum

and

$\frac{1}{\tau_{\text {eff }}}=\frac{0.028}{\tau_{R l}}+\frac{0.018}{\tau_{R t}}$ for $\mathrm{Pb}$ on $\mathrm{Si}$ in vacuum.

The trapping factors are 67 and 56 for $\mathrm{Al}_{2} \mathrm{O}_{3}$ and $\mathrm{Si}$ substrates, respectively, if only the dominant transversewave contribution is taken into account.

Ultrasonic total absorption [40] extrapolation results in values of $\Lambda_{w l}=2100 \AA$ and $\Lambda_{w t}=770 \AA$. From these follow by detailed balance (4) $\tau_{R l}=2.1 \times 10^{-9} \mathrm{~s}$ and $\tau_{R t}=0.79 \times 10^{-10} \mathrm{~s}$ for $\Delta=4 k T$ and $\Delta=1.35 \mathrm{meV}$, i.e. $T=3.92 \mathrm{~K}$, and the intrinsic decay time

$\tau_{R}=0.72 \times 10^{-10} \mathrm{~s}$

or the lifetime $\tau_{R}^{*}=1.45 \times 10^{-10} \mathrm{~s}$, result again with dominant transverse wave contribution. For comparison with experimental and theoretical data cited above we reduce our $\tau_{R}$ to $\tau_{R}^{*}$ at $1.44 \mathrm{~K}$ and obtain: $\tau_{R}^{*}=2.4 \times 10^{-7} \mathrm{~s}$. The rough agreement with the experimental value [38] appears fortuitous, instead the theoretical values $[5,15]$, which are about one order of magnitude below our value, indicate again that the mean free path for transverse phonons is smaller than obtained from the ultrasonic extrapolation. This is in agreement with the phonon mean free path obtained from the data of Kaplan et al. [19] with $\Lambda_{w}=350 \AA$. But disagreement exists with $\tau_{R}^{*}=3.9 \cdot 10^{-9}$ sat $\Delta / k T=4$ as derived from their work. Since, assuming $A_{w}<770 \AA$, all experiments have been performed in Range 2 with 
Table 3. Intrinsic recombination lifetimes $\tau_{B}^{*}$ calculated from $A_{w}$ estimates compared with experimental values and data of $\Delta$ and $N(O)$ used for the calculation

\begin{tabular}{|c|c|c|c|}
\hline & $\mathrm{Al}$ & $\mathrm{Sn}$ & $\mathrm{Pb}$ \\
\hline$\Delta[\mathrm{meV}]$ & 0.20 & 0.59 & 1.35 \\
\hline$N(O)\left[\mathrm{eV}^{-1} \mathrm{~cm}^{-3}\right]$ & $1.75 \times 10^{22 \mathrm{a}}$ & $1.42 \times 10^{22 a}$ & $2.22 \times 10^{22 \mathrm{a}}$ \\
\hline$\Lambda_{w t}[\AA] \quad(\Omega=2 A)$ & $3500^{\mathrm{b}}$ & $700^{\mathrm{c}}$ & $770^{\mathrm{d}}$ \\
\hline$\Lambda_{w t}[\AA] \quad(\Omega=2 \Delta)$ & $7200^{\mathrm{e}}$ & $1600^{\mathrm{f}}$ & $2100^{\mathrm{d}}$ \\
\hline $\begin{array}{l}\tau_{R}^{*}[\mathrm{~s}] \text { calc. from (4) with } \Lambda_{w l, t} \\
(\Delta / k T=4)\end{array}$ & $2.1 \times 10^{-7}$ & $1.27 \times 10^{-9}$ & $1.45 \times 10^{-10}$ \\
\hline $\begin{array}{l}\tau_{R}^{*}[s] \exp \\
(\Delta / k T=4)\end{array}$ & $1.54 \times 10^{-7 \mathrm{~g}}$ & $2.0 \times 10^{-9 h}$ & - \\
\hline
\end{tabular}

References: ${ }^{\mathrm{a}}[46] ;{ }^{\mathrm{b}}[16] ;{ }^{\mathrm{c}}[29] ;{ }^{\mathrm{d}}[40] ;{ }^{\mathrm{e}}[17] ;{ }^{\mathrm{f}}[30] ;{ }^{\mathrm{g}}[18] ;{ }^{\mathrm{h}}[27]$ with a trapping factor of 15.

$d>\Lambda_{w}$, we now discuss $\tau_{\text {eff }}(d)$ according to (2). From the data of Tables 1 and 2 we obtain:

$\tau_{\text {eff }}=1.28 \times 10^{-3} d /[\mathrm{cm}][\mathrm{s}]$

at $\Delta / k T=4$ or $T=3.92 \mathrm{~K}$ for $\mathrm{Pb}$ on $\mathrm{Al}_{2} \mathrm{O}_{3}$ and further $\tau_{\text {eff }}=0.88 \times 10^{-3} d /[\mathrm{cm}][\mathrm{s}]$ for Pbon Si. For $T=1.44 \mathrm{~K}$ and a film of $1250 \AA$ thickness on $\mathrm{Al}_{2} \mathrm{O}_{3}$, this results in $\tau_{\text {eff }}=5.9 \times 10^{-5} \mathrm{~s}$, which exceeds the experiment [38] by two orders of magnitude.

We note that at $1 \mathrm{~K} \tau_{\text {eff }}$ increases to $\tau_{\text {eff }}=9 \times 10^{-3} \mathrm{~s}$ as follows from the exponential temperature dependence. Since the exponential temperature law has not been checked in the measurements [38] we believe that strong overinjection [2] has lowered the experimental value as a consequence of a quasiparticle population highly increased above thermal equilibrium. This is substantiated by phonon pulse experiments [41] using lead junctions as phonon detectors which almost show no temperature dependence of $\tau_{\text {eff }}$ in the range of $1 \mathrm{~K}$ to $2 \mathrm{~K}$. Also in optical excitation [39] the overinjection limit is easily reached. More measurements in lead are to be awaited in order to draw conclusions on the model and also on the question of volume which may be of special importance in this superconductor.

\section{Conclusions and Summary}

The influence of recombination phonon reabsorption and reemission on quasiparticle decay times can be described by an acoustical ray or ballistic phonen propagation model [1] with three characteristic ranges of the thickness dependence of the experimental quasiparticle density decay time $\tau_{\text {eff }}(d)$. In the limit of $d$ small compared to the phonon reabsorption mean free path $A_{w}$ only the limiting angles of total phonon reflection $\varphi_{\max }$ at the film boundaries determine the phonon trapping factor or the ratio $\tau_{\text {eff }} / \tau_{R}$ with $\tau_{R}$ the intrinsic or true quasiparticle decay time without phonon trapping. Experimental data for $\mathrm{Al}$ on $\mathrm{Al}_{2} \mathrm{O}_{3}$ substrates $[14,11,12]$ with different outer surface conditions in this film thickness range scatter between $\tau_{\mathrm{eff}}=\tau_{R}$ and $\tau_{\mathrm{eff}}=7 \cdot \tau_{R}$. The trapping factor of 7 is calculated from the limiting angle of total reflection at the $\mathrm{Al}-\mathrm{Al}_{2} \mathrm{O}_{3}$ boundary without contact to liquid $\mathrm{He}$. Further results are listed in Table 2. For the film thickness range $d>A_{w}$ the model results in a linear increase of $\tau_{\text {eff }}(d)$ in the absence of volume losses, i.e. $\Lambda_{v} \gg \Lambda_{w}$. Experimental data for $\mathrm{Al}$ on $\mathrm{Al}_{2} \mathrm{O}_{3}$ [14] and $\mathrm{Sn}$ on $\mathrm{Si}[9,25]$ agree with the constant of proportionality calculated from the model by use of the phonon and electron densities of state and the phonon escape rate across the film-substrate boundary, as obtained from acoustical data (see Table II). In addition, the quantitative determination of enhanced phonon escape by contact with liquid ${ }^{4} \mathrm{He}$ agrees with studies of the Kapitza resistance anomaly.

So far experiments in the linear $\tau_{\text {eff }}(d)$ range up to $d$-values of $5000 \AA$ for Sn films with trapping factors of 240 have not indicated any influence of volume losses of phonons leading to a saturation of $\tau_{\text {eff }}$ at large film thicknesses $d \gg \Lambda_{w}$. From this follows that in phonon pulse experiments using superconductor tunneling junctions as generators under vacuum conditions all primary recombination phonons are finally radiated into the substrate crystal without losses, i.e. no "thermalisation" of recombination phonons takes place even at film thickness values far exceeding $A_{w}$. Experiments with extremely thick films are of interest with respect to the determination of volume losses and the possibility of $\tau_{R}$ calculations [1].

For the $\tau_{\text {eff }}(d)$ dependence of $\mathrm{Pb}$ films and other film substrate combinations, calculated results are presented in Table 2. 
For $\mathrm{Al}, \mathrm{Sn}$, and $\mathrm{Pb}$ the intrinsic recombination time $\tau_{R}$ is evaluated from the phonon mean path $\Lambda_{w}$ as obtained by the extrapolation of ultrasonic absorption or by direct estimates (see Table 3).

Acknowledgements. We gratefully acknowledge discussions with C. J. Atkins and A. R. Long, and are especially indebted to A. R. Long for privately communicating further experimental data. Financial support granted by the "Deutsche Forschungsgemeinschaft" to part of the experimental work is gratefully acknowledged.

\section{References}

1. W. Eisenmenger, K.Laßmann, H.J.Trumpp, R.Krauß: Appl. Phys. 11, 307 (1976)

2. A.Rothwarf, B. N. Taylor: Phys. Rev. Letters 19, 27 (1967)

3. A. B. Pippard: Proc. R. Soc. A 257, 165 (1960); and Phil. Mag. 46, 1104 (1955)

4. V.M. Bobetic: Phys. Rev. 136 A, 1535 (1964)

5. J.R. Schrieffer, D. M. Ginsberg: Phys. Rev. Lett. 8, 207 (1962)

6. L. Tewordt: Phys. Rev. 127, 371 (1962)

7. K.E. Gray: Phil. Mag. 20, 267 (1969)

8. J.R. Leibowitz: Phys. Rev. 136 A, 22 (1964)

9. H.J.Trumpp, K. Laßmann, W. Eisenmenger: Phys. Lett. 41 A, $431(1972)$

10. W.Eisenmenger: In Fachbericht Physikertagung Berlin 1967, ed. by Deutsche Physikalische Gesellschaft (B.G. Teubner, Stuttgart 1967) p. 88

11. B.I. Miller, A.H. Dayem: Phys. Rev. Lett. 18, 1000 (1967). We used values at higher temperatures, not influenced by overinjection

12. J.L.Levine, S.Y.Hsieh: Phys. Rev. Lett. 20, 994 (1968)

13. K.E.Gray, A.R.Long, C.J.Adkins: Phil. Mag. 20, 273 (1969)

14. A. R. Long: J. Phys. F (Metal Phys.) 3, 2041 (1973) and additional data privately communicated

15. A. Rothwarf, M.Cohen: Phys. Rev. 130, 1401 (1963)

16. A. R. Long: J. Phys. F (Metal Phys.) 3, 2023 (1973)

17. R. David: Philips Res. Repts. 19, 524 (1964)

18. L. N. Smith, J. M. Mochel: Phys. Rev. Lett. 35, 1597 (1976)

19. S. B. Kaplan, C.C.Chi, D. N.Langenberg, J.J.Chang, S.Jafarey, D.J.Scalapino, 1976, to be published see also D. N. Langenberg: In: Low Temperature Physics LT 14, ed. by M. Krusius and H. Vuorio (North Holland/Elsevier, Amsterdam 1975) Vol. 5, p. 223

20. The phonon mean free path is obtained from the phonon lifetime $[19] \tau_{\mathrm{Oph}}(2 \Delta)$ multiplied by the (dominant) transverse sound velocity

21. W.A. Little: Can. J. Phys. 37, 334 (1959); O. Weis: J. Physique 33, C4-49 (1972)

22. H. Kinder: J. Physique 33, C4-21 (1972)

23. M. Welte, K.Lassmann, W.Eisenmenger: J. Physique 33, C4-25 (1972)

24. W.Forkel, M. Welte, W. Eisenmenger: Phys. Rev. Lett. 31, 215 (1973)
25. R.KrauB: Diplomarbeit Universität Stuttgart (1973) unpublished

26. W.Eisenmenger: In Tunneling Phenomena in Solids, ed. by E. Burstein and S. Lundquist (Plenum Press, New York 1969) p. 371

27. G.A.Sai-Halász, C.C.Chi, A. Denenstein, D.N.Langenberg: Phys. Rev. Lett. 33, 215 (1974); see also D. N. Langenberg: In Festungskörperprobleme XIV, Advances in Solid State Physics, ed. by H.J.Queisser (Pergamon, Vieweg, Braunschweig 1974) p. 67

28. W.H. Parker: Sol. State Comm. 15, 1003 (1974)

29. V. Narayanamurti, R.C.Dynes: Phys. Rev. Lett. 27, 410 (1971)

30. J.R.Leibowitz: Phys. Rev. 133 A, 84 (1964)

31. Long [14] compared his experimental result for $\tau_{\text {eff }}^{*}(d)$ with an expression similar to our (2) but without taking account of the longitudinal phonon escape. He found agreement between experiment and theory using a value of $\bar{T}_{t}=0.11$ different from our $\bar{T}_{t}=0.20$ according to Little's [21] work. Further, his calculation differs from ours by using another value for $N(O)$, slightly different sound velocities and an additional factor of 2 in his formula for $\tau_{\text {eff }}^{*}$

32. From (4) with our $N_{T}$ and $N_{\omega T}$ data the $\tau_{R}^{*}$ values of Kaplan et al. [19] result in a $\tau_{w}$ about twice as high as the $\tau_{\text {oph }}$ data given there

33. P.Hu, R.C.Dynes, V. Narayanamurti: Phys. Rev. B 10, 2786 (1974)

34. H. Kinder, W. Dietsche: Phys. Rev. Lett. 33, 578 (1974)

35. C.J.Guo, H. J. Maris: Phys. Rev. Lett. 29, 855 (1972)

36. T. J. B. Swanenburg, J. Wolter: Phys. Rev. Lett. 31, 693 (1973)

37. W. Eisenmenger, A. H. Dayem: Phys, Rev. Lett. 18, 125 (1967)

38. D. M. Ginsberg: Phys. Rev. Lett. 8, 204 (1962)

39. W.H. Parker, W.D.Williams: Phys. Rev. Lett. 29, 924 (1972)

40. W.P. Mason, H.E.Bömmel: J. Acoust. Soc. Am. 28, 930 (1956)

41. R. Rolcke: private communication

42. L. Bergmann: Der Ultraschall, 6. Aufl. (S. Hirzel, Stuttgart 1954). The sound velocities used are those for polycrystalline material corresponding to the polycrystalline film structure

43. C. Kittel: Introduction to Solid State Physics, 3rd ed. (John Wiley \& Sons, New York 1967) p. 122.

The sound velocities for $\mathrm{Pb}$ are weighted averages of the values from [43] for different crystal orientations. More significant average values especially for the dominant transverse modes can be obtained from low temperature lattice specific heat data. For $\mathrm{Pb}$ with $\theta=88 \mathrm{~K}$ this results in $c_{\mathrm{t}}=0.8 \times 10^{5} \mathrm{~cm} \mathrm{~s}^{-1}$. For $\mathrm{Al}$ and $\mathrm{Sn}$ the specific heat evaluation agrees essentially with the listed data

44. O. Weis: private communication

45. Data labelled (c) on $\bar{T}_{t}$ and $\bar{T}_{l}$ are obtained approximating $\bar{T}$ by $\bar{T}=T_{\perp} \sin ^{2} \varphi_{\max }, T_{\perp}$ being the normal incidence transmission factor. Data labelled (a) and (b) are taken from literature as indicated

46. T.P.Sheahen: Phys. Rev. 149, 370 (1966)

Note added in proof. The lifetimes in [18] have to be multiplied by a factor of 2.84. (Private communication by L.N.Smith) 